\title{
Electrophysiological Aspects in Idiopathic Acute Canine Polyradiculoneuritis
}

\author{
Gabriela-Dumitrița STANCIU ${ }^{1}$, Mihai MUSTEAȚ $\breve{A}^{1}$, Mihaela ARMAȘU ${ }^{1}$, Paula Maria SAFTENCU ${ }^{1}$, \\ Gheorghe SOLCAN ${ }^{1 *}$
}

${ }^{1}$ University of Agricultural Science and Veterinary Medicine "Ion Ionescu de la Brad" of Iasi
Faculty of Veterinary Medicine, Department of Internal Medicine / Neurology
Address: 8, Mihail Sadoveanu Alley, 700489, Iasi, Romania
${ }^{*}$ corresponding author: gsolcan@uaiasi.ro

Bulletin UASVM Veterinary Medicine 71(2) / 2014,

Print ISSN 1843-5270; Electronic ISSN 1843-5378

DOI: 10.15835/buasvmcn-vm: 10276

\begin{abstract}
Polyradiculoneuritis is an acute-onset, monophasic, immune-mediated polyneuropathy that often follows an antecedent infection. It is more common in dogs and rare in cats. The most frequent type is Coonhound paralysis. This is similar to Landry-Guillain-Barré syndrome in humans and seems to be secondary to a raccoon bite, probably due to some factor in the saliva. However, it can also occur without any interaction with a raccoon - idiopathic acute polyradiculoneuritis (IAPR). This rare disease is characterized by acute progressive flaccid quadriparesis and hyporeflexia, facial paralysis and laryngeal weakness.

The purpose of the current study was to describe electrodiagnostic recording tests and analyse the electrophysiological abnormalities recorded in dogs with idiopathic acute polyradiculoneuritis.

Material and methods: Electrophysiological investigations were performed on 5 dogs with suspected acute canine polyradiculoneuropathy of different severity; mixed breeds, between five and eleven years old.

The electrophysiological tests were performed using the Neuropack S, MEB 9400 K Electrodiagnostic System (Nihon Kohden) in the Electromyography (EMG) program.

Results and discussions: The most reliable electrophysiological indicators of idiopathic polyradiculoneuritis were electromyography changes, nerve conduction studies showed marked dispersion and prolonged latency of F-waves, indicative of slowed conduction in the ventral roots. These findings suggest that IAPR represents a peripheral motor axonopathy, with demyelination and axonal involvement also occurring in ventral nerve roots.

Conclusions: IAPR the demyelinating subtype is characterized by emphasizing the damage of the peripheral motoneuron pericarion translated by increased $\mathrm{F}$ wave latencies accompanied by nonspecific electromyographic changes and low VCN; but repetitive stimulation with physiological values.
\end{abstract}

Keywords: acute demyelinating polyradiculoneuropathy, electrophysiology, dog, idiopathic acute polyradiculoneuritis

\section{INTRODUCTION}

Polyradiculoneuritis is an acute-onset, monophasic, immune-mediated polyneuropathy of multiple nerve roots and peripheral nerves; that often follows an antecedent infection. The most frequent type is Coonhound paralysis. This is similar to Landry-Guillain-Barré syndrome in humans and seems to be secondary to a raccoon bite, probably due to some factor in the saliva. However, it can also occur without any interaction with a raccoon - idiopathic acute polyradiculoneuritis (IAPR). This rare disease is characterized by acute progressive flaccid quadriparesis and hyporeflexia, facial paralysis and laryngeal weakness (Cuddon, 2002; Dumitru, 2002).

The typical patient with IAPR, which in most cases will be acute inflammatory demyelinating polyradiculoneuropathy (AIDP) and acute motor axonal neuropathy (McKhann et al., 1993; Hadden et al., 1998), 2-4 weeks following a relatively benign respiratory or gastrointestinal illness (Kimura, 2001). Initially, there is a short-strided gait in pelvic 
limbs that progresses within 1-2 days to flaccid tetraparesis or tetraplegiaand, in somecasesaffected cranial nerves. Death from respiratory paralysis can occur in severe cases. Spinal cord reflexes are weak to absent, and severe muscle atrophy is evident within 10-14 days. Pain perception is intact, and some dogs may appear hyperesthetic. Mentation and appetite are not affected. Urination, defecation, and tail movement usually remain normal (Cuddon, 2002; Molin, 2011).

IAPR is considered to be a post infectious, immune-mediated disease targeting peripheral nerves. Up to two thirds of patients report an antecedent bacterial or viral illness prior to the onset of neurologic symptoms (Kimura, 2001). Respiratory infections are most frequently reported, followed by gastrointestinal infections. Infections with Haemophilus influenzae, Borrelia burgdorferi, para-influenza virus type 1, influenza $A$ virus, influenza $B$ virus, adenovirus, and herpes simplex virus have been demonstrated in patients with IAPR (Dumitru, 2002; Cuddon, 2002). Chetboul (1989) also reported a case of postvaccinal polyradiculoneuritis. Tumor necrosis factor-alpha polymorphisms with increased expression are associated with many autoimmune and inflammatory diseases, and may increase susceptibility to axonal AIPR subtypes (Dumitru, 2002). However, the role of these polymorphisms in acute idiopathic polyradiculoneuritis remains unclear and warrants further investigation.

Most affected animals begin to improve spontaneously within 3 weeks, with complete recovery by 2-6 months. Animals with severe signs and marked muscle atrophy may never recover completely. Relapses may occur, especially in hunting dogs that frequently encounter raccoons (Thomas, 2013).

The diagnosis protocol of acute flaccid tetraparesis a patient appeals to a large number of tests as long as clinical signs are identical to a pathology of peripheral nerve, neuromuscular junction and muscle.

Combining electrophysiology tests provide sufficient evidence for discrimination of the mentioned conditions in real time. They may also indicate further testing (muscle biopsy, nerve biopsy, testing of antibodies anti-acetylcholine receptor) - more expensive.

In this study we describe the electrodiagnostic recording tests performed and we will analyse the electrophysiological abnormalities recorded in dogs with idiopathic acute polyradiculoneuritis.

\section{MATERIAL AND METHODS}

Electrophysiological investigations were performed on 5 dogs (mixed breeds, between five and eleven years old), diagnosed with acute canine polyradiculoneuropathy of different severity, presented to Neurology Service of Faculty of Veterinary Medicine Iasi.

The diagnosis was made on the basis on clinical examination, blood, and cerebrospinal fluid (CSF) analysis and acetylcholine receptor antibody test. Each patient was tested by electrodiagnostic: electromyography (EMG), repetitive nerve stimulation (RNS), motor nerve conduction velocity (MNCV) and late wave testing: F-waves.

All patients were sedated before electrodiagnostic tests using medetomidine hydrochloride (Domitor, Pfizer) $0.03 \mathrm{mg} / \mathrm{kg}$ inj. i.m.

The electrophisiological tests were performed with the animal in lateral recumbency, using the Neuropack S, MEB 9400 K Electrodiagnostic System (Nihon Kohden) in the EMG program. The area investigated was trimmed, degreased with alcohol and Skin Pure (Nihon Kohden), and surface electrodes were attached with special gel (Elefix, Nihon Kohden).

The following muscles were examined: biceps femoral, interdigital and quadriceps. During the electromyography a concentric bipolar needle was introduced percutaneously in different regions of the muscle at different depths. To obtain a representative sample the muscle was tested in 5 to 10 regions. The reference electrode was placed at the motor point of every muscle.

MNCV testing involved the placement of a pair of recording surface electrodes near a muscle innervated by the nerve of interest and at least two pairs of stimulating electrodes along the course of that nerve. A ground electrode was used to minimize interference. The active recording electrode was placed over the motor point of the muscle and the reference electrode was located several centimetres distally. Tibial and radial nerves were examined.

Repetitive nerve stimulation (RNS) is "the technique of repeated supramaximal stimulation of a nerve while recording $M$ waves from muscles innervated by the nerve" (AAEE) (Cuddon, 2003). Recording technique was similar to that previously 
described for MNCV but trains of stimuli at various frequencies are employed. Frequencies tested generally range from $0.5 \mathrm{~Hz}$ to $50 \mathrm{~Hz}$.

The $\mathrm{F}$ wave uses supramaximal stimulation of a motor nerve and records compound muscle action potentials from a muscle supplied by that nerve, along the most proximal segment. For $F$ wave study, a strong electrical stimulus (supramaximal stimulation) was applied to the skin surface above the distal portion of a nerve so that the impulse travels both distally (towards the muscle fibre) and proximally (back to the motor neurons of the spinal cord). Median, ulnar, peroneal and tibial nerves were tested.

\section{RESULTS AND DISCUSSIONS}

Neurological examination findings was characterized by generalized hyporeflexia to areflexia, hypoatonia to atonia. The pelvic limbs were more severely affected than thoracic limbs and the signs were usually symmetrical. Aphonia or dysphonia was encountered in four dogs. Pain sensation was intact. However, motor dysfunction was always predominant. Appetite and water consumption, urination and defecation were normal.

The blood examination of all the patients revealed high levels for AST and alkaline phosphatase. Acetylcholine receptor antibody was negative in all tested patients eliminating hypothesis of a myasthenic syndrome. LCR collected from the lumbar subarachnoid space showed increased protein (less than $50 \mathrm{mg} / \mathrm{dl}$ ) with a normal cell count.

The most reliable electrophysiological indicators of idiopathic polyradiculoneuritis were electromyography changes (occurring in 100\% of affected dogs). Electromyography was characterized by positive sharp waves, complex repetitive discharges and mild fibrillation potentials (fig. 1, 2 and 3 ). This changes indicate a nerve disease with

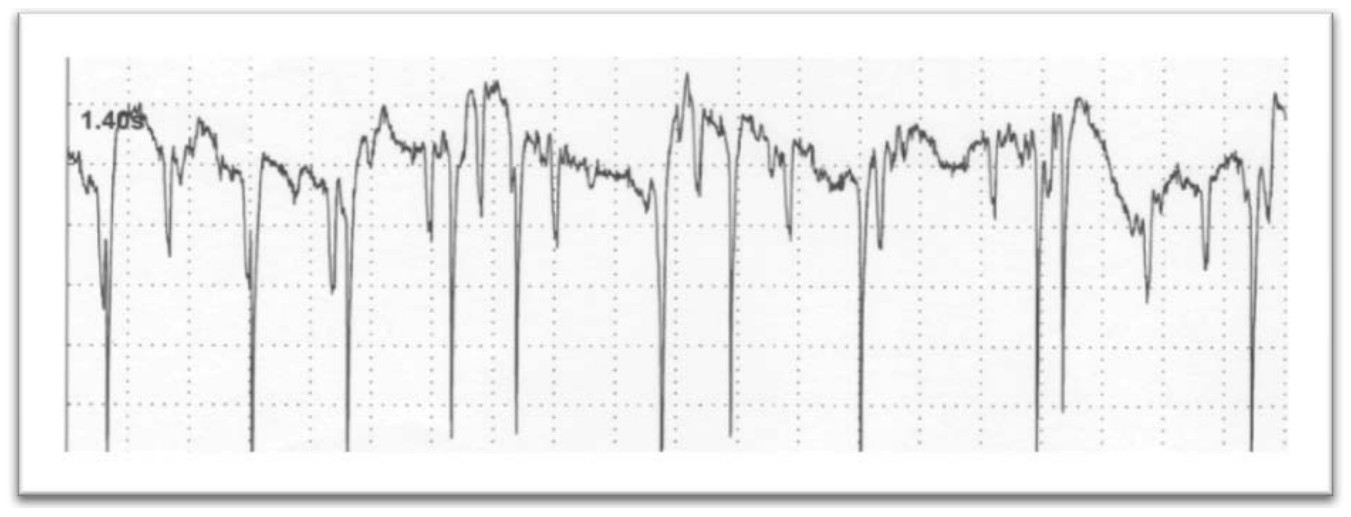

Fig. 1. Positive sharps waves $(100 \mu \mathrm{V} / \mathrm{div} ; 10 \mathrm{msec} / \mathrm{div})$ recording in a six-year-old Pekingese male dog. Right muscle biceps femoris

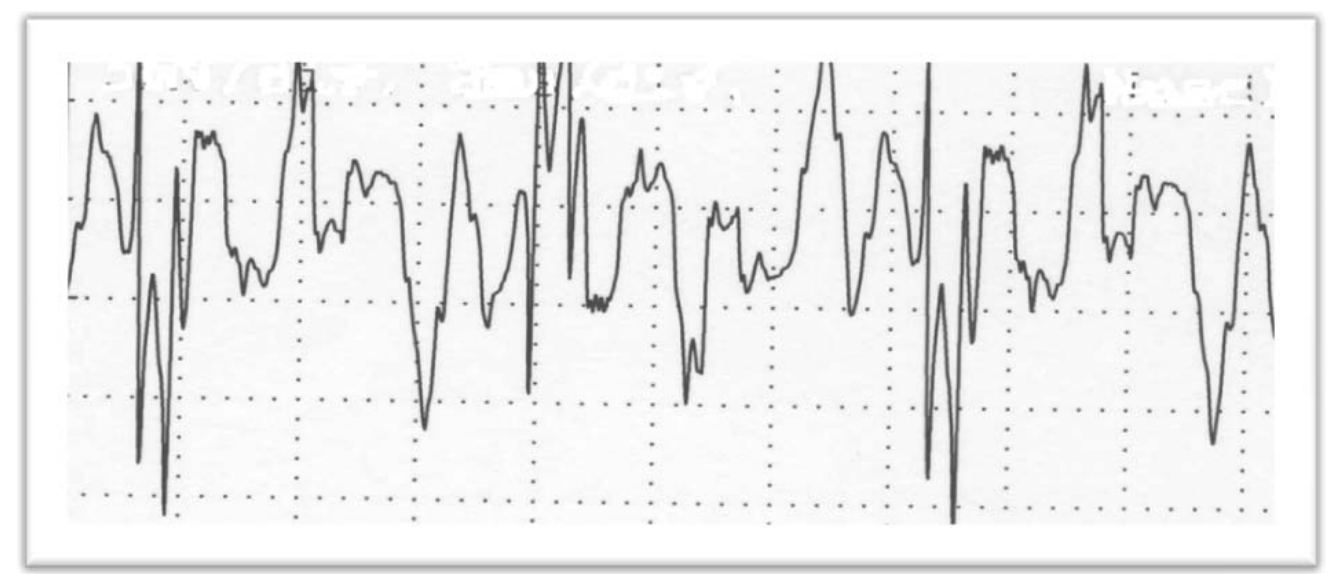

Fig. 2. Complex repetitive discharges - Muscle quadriceps Caniche, female, 8 years old - acute idiopathic polyradiculoneuritis 
preservation of the integrity and functionality of the neuromuscular junction and of the innervated muscles (fig. 4).

Positive sharp wave represent a spontaneous action potential of a single myofiber due to destabilization of the sarcolemma membrane (Cuddon et al., 2003).

Complex repetitive discharges are polyphasic, repetitive waveforms representing spontaneous discharge of multiple myofibers (Cuddon et al., 2003).

In fig. 4 are shown the results of the eight-yearold Caniche, female. There is a physiological 5.6\% reduction in the amplitudes of the third cMAP compared to the first cMAP. A loss in amplitude with $10 \%$ is considered physiological, suggesting that there is no disturbance in neuromuscular transmission (Dumitru, 2002).

Electrophysiological study suggests a simultaneous impairment of ventral roots and motor pe- ripheral nerves. Neuromuscular junctions were intact eliminating the hypothesis of a myasthenic syndrome or botulism.

Electrophysiology tests were then conducted to identify the subtypes of polyradiculoneuritis in wich the studied pacient fall. The classification is based on nerve-conduction studies (Fig. 5).

There is a notable difference in the geographic distribution of subtypes of the syndrome. In Europe and North America, the demyelinating IAPR syndrome ranges up to $90 \%$ of cases, $29 \%$ whereas in China, Japan, Bangladesh, and Mexico, the frequency of the axonal IAPR syndrome ranges from $30 \%$ to $65 \%$ and the frequency of the demyelinating IAPR syndrome ranges from $22 \%$ to 46\% (McKhann et al., 1993; Hadden et al., 1998).

Electroneurography (fig. 5) showed a marked dispersion with polyphasic action potentials, a decrease in conduction velocity $(40 \mathrm{~m} / \mathrm{s}$ compared to the refference values of $70 \mathrm{~m} / \mathrm{s}$ ) and a smaller

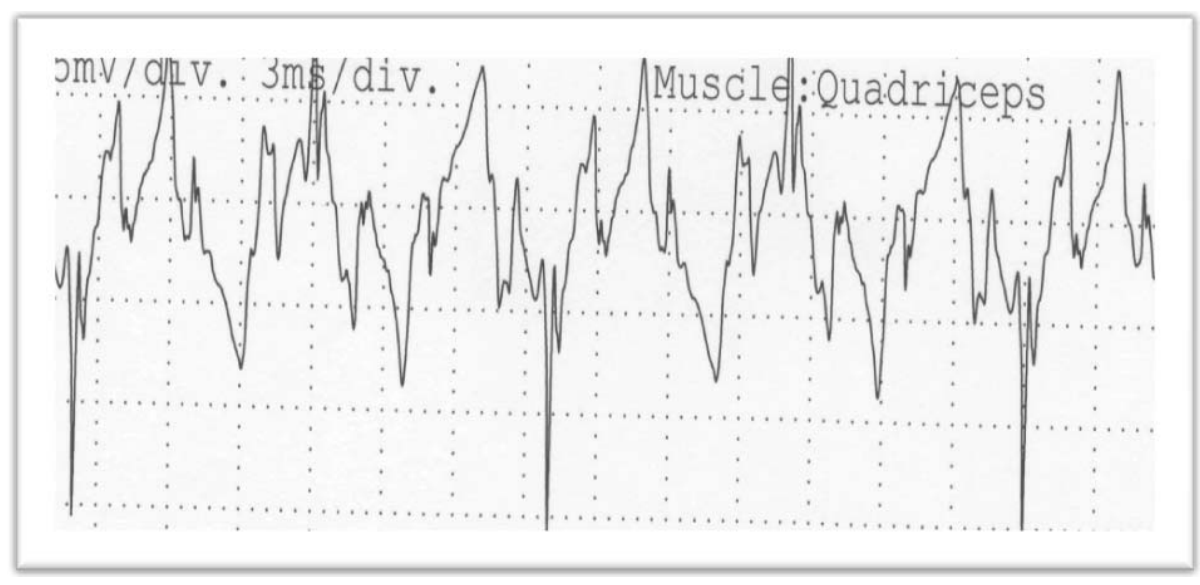

Fig. 3. Fibrillation potentials (severe) with very occasional positive sharp waves Caniche, female, 8 years old - Muscle quadriceps

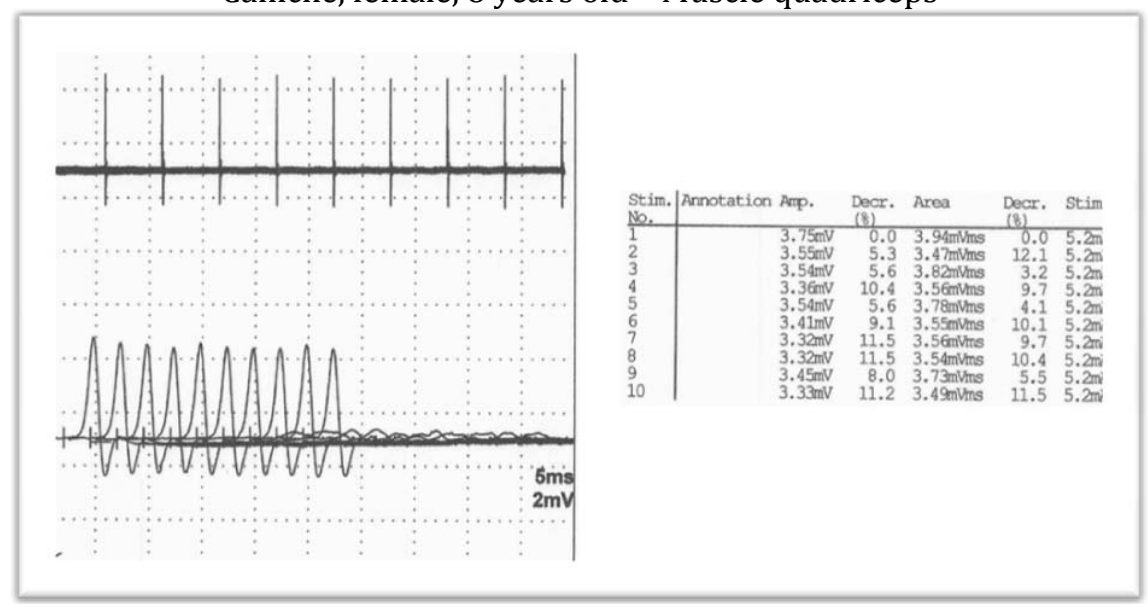

Fig. 4. Repetitive nerve stimulation tests the fatigability of the synaptic transmission 
amplitude especially in the distal portion of the nerve.

The decrease of nerve conduction velocity, with a significant reduction in the amplitude of $M$ potential suggests demyelination. The latter can induce, as a result, a lower amplitude of the VCN and M polyphase potential, due to different degrees of myelination of fibers (Dumitru, 2002)

The reduction of the amplitude of the $M$ proximal potential towards the distal M potential, which doesn't register any change, associated with its poliphase formats corresponds to a conduction block. Conduction block occurred due nerve segmental demyelination with a higher than two internodes length (Jaggy, 2007).

According to the VCN, the patients in the study had acute idiopathic polyradiculoneuritis the demyelinating subtype.

The diagnosis of acute idiopathic polyradiculoneuritis demyelinating subtype was confirmed by the favourable evolution of the disease with complete functional recovery in about six weeks, using just physiotherapy and sustaining.

Analysing the data we found that in all cases of IAPR, electrophysiological test were characterized by maintaining physiological aspects in neuromus-

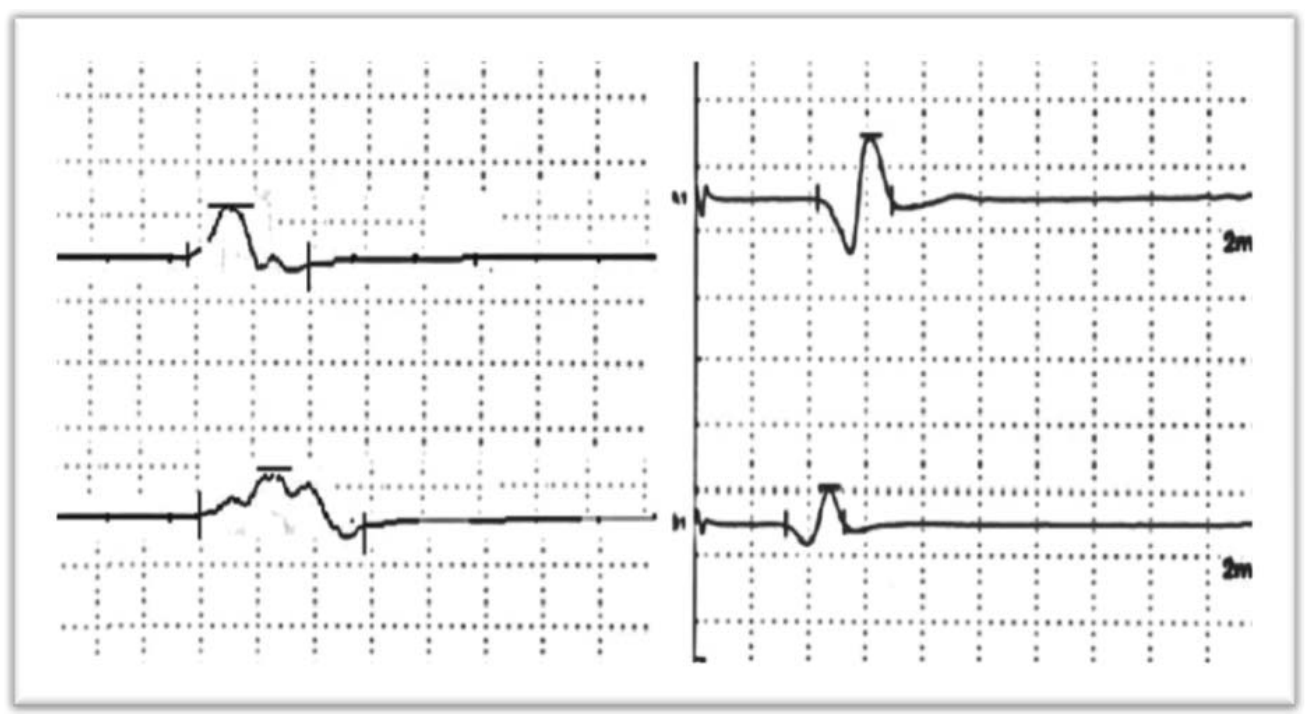

Fig. 5. Nerve conduction velocity - Left tibial nerve. Eleven-year-old, female, Westie.

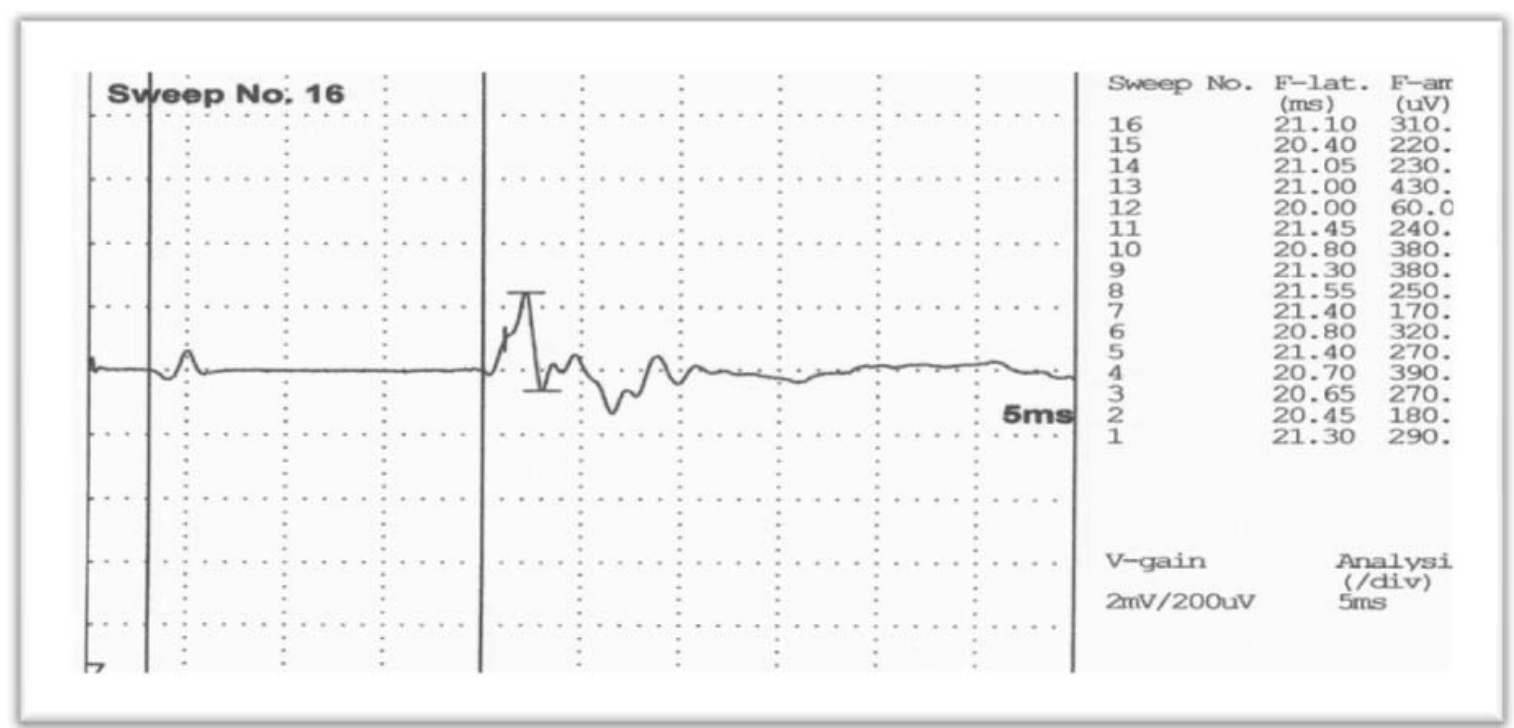

Fig. 6. Tibial nerve F waves, Westie, female, 11 years old. Latency of $20 \mathrm{~m} / \mathrm{s}$ reveal an F wave relationship significantly above the normal value (maximum $10 \mathrm{~m} / \mathrm{s}$ ), which indicates severe disease of the nerve roots and the proximal segments of the nerve. 
cular junctions with neurogenic alterations. The electrodiagnostic allow us to establish a patient's clinical prognosis.

\section{CONCLUSIONS}

Acute idiopathic polyradiculoneuritis the demyelinating subtype is characterized by emphasizing the damage of the peripheral motoneuron pericarion translated by increased $\mathrm{F}$ wave latencies accompanied by nonspecific electromyographic changes (positive sharp waves, complex repetitive discharges and mild fibrillation potentials) and low VCN; but repetitive stimulation with physiological values.

Acknowledgment - this paper was published under the frame of European Social Fund, Human Resources Development Operational Programme 2007-2013, projectno.POSDRU/159/1.5/S/132765.

\section{REFERENCES}

1. Chetboul V., (1989). Cas clinique: polyradiculonévrite post-vaccinale. Point Vét., 21(124):743-745.

2. Cuddon PA., (2002). Electrodiagnosis in Veterinary Neurology: Electromyography, Nerve Conduction Studies and Evoked Responses. Loveland, CO, p. 111-134.

3. Cuddon P.A., Michelle Murray, Karl Kraus (2003). Textbook of Small Animal Surgery. Electrodiagnosis. Ed. Douglas H. Slatter, Elsevier Health Sciences, p. 108-213.
4. Cummings JF, Haas DC (1966). Coonhound paralysis. An acute idiopathic polyradiculoneuritis in dogs resembling the Landry-Guillain-Barré syndrome. J Neurol Sci 4(1):5181.

5. Cummings JF., (1992). Canine Inflammatory Polyneuropathies. In: Kirk RW Bonagura JD, eds. Current Veterinary Therapy XI Small animal Practice Philadelphia: WB Saunders, p. 1034-1035.

6. Dumitru D., (2002). Electrodiagnostic Medicine. 2nd edition, Hanley and Belfus, Inc. Philadelphia, PA, p.234245.

7. Hadden RDM, Cornblath DR, Hughes RAC, (1998). Electrophysiological classification of Guillain-Barré syndrome: clinical associations and outcome. Ann Neurol 44:780-8.

8. Gehring R, Eggars B (2001). Suspected post-vaccinal acute polyradiculoneuritis in a puppy. Journal of the South African Veterinary Association 72(2): 96.

9. Kimura J., (2001). Electrodiagnosis in Diseases of Muscle and Nerve: Principles and Practices 3rd edition. Oxford University Press, New York, p. 123-342.

10. McKhann GM, Cornblath DR, Griffin JW, Ho TW, Li CY, Jiang Z, Wu HS, Zhaori G, Liu Y, Jou L (1993). Acute motor axonal neuropathy: a frequent cause of acute flaccid paralysis in China. Ann Neurol; 33 (4):333-42.

11. Molín J, Márquez M, Raurell X, Matiasek K, Ferrer I, Pumarola M, (2011). Acute clinical onset chronic inflammatory demyelinating polyneuropathy in a dog. Muscle Nerve, 44(3):441-444. 